УДК 517.55

\title{
Correctness of a Two-dimensional Cauchy Problem for a Polynomial Difference Operator with Constant Coefficients
}

\author{
Marina S. Apanovich* \\ Krasnoyarsk State Medical University \\ Partizana Zheleznyaka, 1, Krasnoyarsk, 660022 \\ Evgeny K. Leinartas ${ }^{\dagger}$ \\ Institute of Mathematics and Computer Science \\ Siberian Federal University \\ Svobodny, 79, Krasnoyarsk, 660041
}

Russia

Received 16.11.2016, received in revised form 08.02.2017, accepted 20.02.2017

The correctness of Cauchy problem for a polynomial difference operator is studied. An easily verifiable sufficient condition for correctness of a two-dimensional Cauchy problem for an operator with constant coefficients is proved.

Keywords: polynomial difference operator, Cauchy problem, correctness.

DOI: $10.17516 / 1997-1397-2017-10-2-199-205$.

\section{Introduction}

The theory of linear differential equations is used in various areas of mathematics, and in onedimensional case is largely completed [1]. The situation is much more difficult for $n>1$, and there is no general description of the space of solutions to the difference equation even for operator with constant coefficients. One way to describe is to ask additional (initial, boundary) conditions on the solution of the equation that ensure uniqueness. For multidimensional difference equations in the positive orthant of the integer lattice various options for setting these conditions (a Cauchy problem) have been considered in $[2,3]$ in connection with applications to the combinatorial enumeration analysis.

Another important source of difference equations is discretization of differential ones. In this case the form of the domain of a solution to a difference equation and the type of additional restrictions on the solutions are determined by the initial differential problem and the chosen approximation method. The resulting problem is called a difference scheme and the question of the existence and uniqueness of solutions is central in the corresponding theory [4-6]).

Another important question is the question of stability of a difference equation. For $n=1$ and constant coefficients the stability is investigated in the framework of the theory of discrete dynamical systems (see, e.g. [7]), and it is completely determined by the roots of the characteristic polynomial, namely, if they all lie in the unit circle. Stability of two-dimensional digital recursive filter has been studied in [8]. In the monograph [9] the question of stability has been studied for a two-layer homogeneous linear difference scheme with constant coefficients. Stability of multilayer

\footnotetext{
*rogozina.marina@mail.ru

$\dagger$ lein@mail.ru

(c) Siberian Federal University. All rights reserved
} 
homogeneous difference schemes has been investigated in papers $[6,10,11]$ by using methods of the theory of amoebas of algebraic hypersurfaces, in this case a formula representing a solution to a Cauchy problem via its fundamental solution has been obtained.

A difference analog of the boundary value problem of Hörmander for a polynomial differential operator has been studied in [12] in two-dimensional case and in [13] for arbitrary number of variables. In [13] we have studied solvability of difference equations with initial-boundary Riquiertype conditions; in terms of the theory of difference schemes they are multilayer implicit difference schemes. In [14] an easily verifiable sufficient condition for correctness of a Cauchy problem for a polynomial difference operator with constant coefficients whose characteristic polynomial is homogeneous has been obtained.

In this paper we investigate correctness of a Cauchy problem for some polynomial difference operators that often appear in the theory of difference schemes.

Let us introduce necessary notation and definitions. Denote by $\mathbb{Z}$ the set of integers, by $\mathbb{Z}^{2}=\mathbb{Z} \times \mathbb{Z}$ the two-dimensional integer lattice and by $\mathbb{Z}_{+}^{2}$ the subset of the lattice consisting of points with non-negative integer coordinates. Define the shift operators $\delta_{1}$ and $\delta_{2}$ with respect to $x$ and $y$ correspondingly as $\delta_{1} f(x, y)=f(x+1, y)$ and $\delta_{2} f(x, y)=f(x, y+1)$.

Consider a linear polynomial difference operator of the form

$$
P\left(\delta_{1}, \delta_{2}\right)=\sum_{j=0}^{m} \sum_{i=0}^{k} c_{i j} \delta_{1}^{i} \delta_{2}^{j}
$$

where $c_{i j}$ are constant coefficients. The polynomial $P(z, w)=\sum_{j=0}^{m} \sum_{i=0}^{k} c_{i j} z^{i} w^{j}$ is called the characteristic polynomial of the operator (1), its degree $\operatorname{deg} P_{w}=m$ with respect to $w$ is called the order of polynomial difference operator $P\left(\delta_{1}, \delta_{2}\right)$ and is denoted by $n=\max _{j}\left\{k_{j}\right\}$.

Consider the strip $\Pi_{K}=\left\{(x, y) \in \mathbb{R}^{2}, 0 \leqslant x \leqslant K-1, y>0\right\}$ of width $K$ in the positive quadrant of the plane $\mathbb{R}^{2}$. Fix $\beta$ such that $c_{\beta m} \neq 0$ and consider the strip $\Pi_{K, \beta}=\left\{(x, y) \in \mathbb{R}_{+}^{2}\right.$ : $0 \leqslant x-\beta \leqslant K-k-1, y>m-1\}$. Denote $L_{K, \beta}=\Pi_{K} \backslash \Pi_{K, \beta}$ and formulate the following problem:

find a solution to the difference equation

$$
P\left(\delta_{1}, \delta_{2}\right) f(x, y)=g(x, y),(x, y) \in \Pi_{K} \cap \mathbb{Z}^{2},
$$

satisfying the condition

$$
f(x, y)=\varphi(x, y),(x, y) \in L_{K, \beta} \cap \mathbb{Z}^{2},
$$

where $g(x, y)$ and $\varphi(x, y)$ are given functions of integer arguments.

The problem (2)-(3) is called the Cauchy problem for the polynomial difference operator $P\left(\delta_{1}, \delta_{2}\right)$.

As an example, we consider the first boundary value problem for the heat transfer equation [4]:

$$
\left\{\begin{array}{l}
\frac{\partial u}{\partial t}=\frac{\partial^{2} u}{\partial t^{2}}, t>0,0<x<l \\
u(0, t)=u_{1}(t), u(l, t)=u_{2}(t) \\
u(x, 0)=u_{0}(x)
\end{array}\right.
$$

and its approximation by the six-point difference (two-layer) parametric scheme of the form

$$
\frac{u_{i}^{m+1}-u_{i}^{m}}{\tau}=\sigma \frac{u_{i+1}^{m+1}-2 u_{i}^{m+1}+u_{i-1}^{m+1}}{h^{2}}+(1-\sigma) \frac{u_{i+1}^{m}-2 u_{i}^{m}+u_{i-1}^{m}}{h^{2}}, i=1,2, \ldots, N-1,
$$




$$
u_{0}^{m}=u_{1}\left(t_{m}\right), u_{N}^{m}=u_{2}\left(t_{m}\right), u_{i}^{0}=u_{0}\left(x_{i}\right), m=0,1, \ldots,
$$

where $0 \leqslant \sigma \leqslant 1$ is an arbitrary real parameter.

Consider a purely implicit scheme, i.e. $\sigma=1$. Then (5) can be rewritten in the form $\frac{u_{i}^{m+1}-u_{i}^{m}}{\tau}=\frac{u_{i+1}^{m+1}-2 u_{i}^{m+1}+u_{i-1}^{m+1}}{h^{2}}$. Let $\gamma=\frac{\tau}{h^{2}}$, then $u_{i}^{m+1}-\frac{1}{1+2 \gamma} u_{i}^{m}-\frac{\gamma}{1+2 \gamma} u_{i+1}^{m+1}-$ $\frac{\gamma}{1+2 \gamma} u_{i-1}^{m+1}=0$. If we denote $a=-\frac{\gamma}{1+2 \gamma}$ then in our notation $c_{01}=c_{21}=a, c_{11}=1$, and the difference operator will have the form $P\left(\delta_{1}, \delta_{2}\right)=a \delta_{2}+\delta_{1} \delta_{2}+a \delta_{1}^{2} \delta_{2}+(2 a+1) \delta_{1}$.

If we reassign $u_{i}^{m}=f(i, m)$, the Cauchy problem (5)-(6) for this scheme takes the form

$$
\begin{gathered}
P\left(\delta_{1}, \delta_{2}\right) f(x, y)=0,0<x<K-1, y=0,1, \ldots, \\
\varphi(x, y)=\left\{\begin{array}{l}
f(0, y), y=0,1, \ldots, \\
f(K, y), y=0,1, \ldots, \\
f(x, 0), x=1,2, \ldots, K-1 .
\end{array}\right.
\end{gathered}
$$

For the function $s: X \rightarrow \mathbb{C}$ we denote $\|s\|_{\infty}=\sup _{X}|s(x)|$.

We say that the problem of the form (2)-(3) for a polynomial difference operator $P\left(\delta_{1}, \delta_{2}\right)$ is correct (well posed), if the following conditions are satisfied:

a) the problem is uniquely solvable, i.e. a solution exists and is unique for any initial data $\varphi(x, y)$ and right hand side $g(x, y)$;

b) there is a constant $M>0$ such that for any $\varphi(x, y)$ and $g(x, y)$ for the norm of the corresponding solution $f(x, y)$ of the problem (2)-(3) there is an estimate

$$
\|f(x, y)\|_{\infty} \leqslant M\left(\|g(x, y)\|_{\infty}+\|\varphi(x, y)\|_{\infty}\right) .
$$

Note that if condition b) is fulfilled the difference operator $P\left(\delta_{1}, \delta_{2}\right)$ it called stable.

Thus, the difference problem (2)-(3) is correct (well posed) if for any initial data $\varphi(x, y)$ and right hand side $g(x, y)$ it is uniquely solvable and stable.

Let us give an easily verifiable condition for correctness of the problem (2)-(3).

Theorem 0.1. If the coefficients of a polynomial difference operator (1) satisfy

$$
\left|c_{\beta m}\right| \geqslant \sum_{(i, j) \neq(\beta, m)}\left|c_{i j}\right|,
$$

then (2)-(3) is uniquely solvable and stable, i.e. the problem (2)-(3) is correct.

\section{The proof of correctness of (2)-(3)}

To prove Theorem 1 we need some concepts and facts from the theory of systems of linear equations with infinite number of unknowns.

The system (2)-(3) is an infinite system of equations with the unknowns $f(x, y),(x, y) \in$ $\Pi_{K} \cap \mathbb{Z}^{2}$. An important feature of this system is that each equation contains a finite number of unknowns. It is known (see [15, Lemma 6.3.7]) that this system is consistent if and only if so is every finite collection of these equations. To study the question of conditions on the operator $P\left(\delta_{1}, \delta_{2}\right)$ under which the problem (2)-(3) is correct, we first order the equations of this system so that at each step the system containes all the equations of the previous step. 
Fix $p$ such that $p \geqslant m$ and consider the rectangle $\Pi_{K}^{p}=\{(x, y): 0 \leqslant x \leqslant K-1,0 \leqslant y \leqslant p\}$. Index the unknowns by the elements of $\Pi_{K}^{p}$ in the lexicographic order. Index also the equations by the elements of two sets $\Pi_{K, \beta}^{p}=\{(x, y): 0 \leqslant x \leqslant K-k-1,0 \leqslant y \leqslant p-m\}$ and $L_{K, \beta}^{p}=\Pi_{K}^{p} \backslash\left\{(\beta, m)+\Pi_{K, \beta}^{p}\right\}$. Since $L_{K, \beta}^{p} \cup\left\{(\beta, m)+\Pi_{K, \beta}^{p}\right\}=\Pi_{K}^{p}$, assign to the elements of $L_{K, \beta}^{p}$ the same indices with which they appear in $\Pi_{K}^{p}$, and to the elements $(x, y)$ of $\Pi_{K, \beta}^{p}$ the indices of $(\beta, m)+(x, y)$ in $\Pi_{K}^{p}$.

We obtain a system of equations for the unknown $f(x, y),(x, y) \in \Pi_{K}^{p}$, of the form

$$
\begin{gathered}
P\left(\delta_{1}, \delta_{2}\right) f(x, y)=g(x, y),(x, y) \in \Pi_{K, \beta}^{p} \cap \mathbb{Z}^{2}, \\
f(x, y)=\varphi\left(\mu_{1}, \mu_{2}\right),\left(\mu_{1}, \mu_{2}\right) \in L_{K, \beta}^{p} \cap \mathbb{Z}^{2} .
\end{gathered}
$$

The number of equations \# $\left(L_{K, \beta}^{p} \sqcup \Pi_{K, \beta}^{p}\right)$ in this system equals the number of unknowns $\# \Pi_{K}^{p}$. The symbol " $\sqcup$ " denotes a disjoint union.

Denote by $A_{p}$ the matrix of the system of equations (9)-(10) and by $\operatorname{det} A_{p}$ its determinant. Example. Consider the difference operator

$$
P\left(\delta_{1}, \delta_{2}\right)=c_{21} \delta_{1}^{2} \delta_{2}+c_{11} \delta_{1} \delta_{2}+c_{01} \delta_{2}+c_{20} \delta_{1}^{2}+c_{10} \delta_{1}+c_{00},
$$

where $m=1, b=2, \beta=1$. Let $K=4, p=2$, then the system of equations (9)-(10) takes the form

$$
\begin{gathered}
c_{21} f(x+2, y+1)+c_{11} f(x+1, y+1)+c_{01} f(x, y+1)+c_{20} f(x+2, y)+ \\
+c_{10} f(x+1, y)+c_{00} f(x, y)=g(x, y),(x, y) \in \Pi_{4,(1,1)}^{2}, \\
f(x, y)=\varphi\left(\mu_{1}, \mu_{2}\right),\left(\mu_{1}, \mu_{2}\right) \in L_{4,(1,1)}^{2},
\end{gathered}
$$

with unknowns $f\left(y_{1}, y_{2}\right)$,

$$
\left(y_{1}, y_{2}\right) \in \Pi_{4}^{2}=\{(0,0),(1,0),(2,0),(3,0),(0,1),(1,1),(2,1),(3,1),(0,2),(1,2),(2,2),(3,2)\} .
$$

The equations in $\left(9^{\prime}\right)$ are indexed by the elements of

$$
\Pi_{4,(1,1)}^{2}=\{(\overline{0}, \overline{0}),(\overline{1}, \overline{0}),(\overline{0}, \overline{1}),(\overline{1}, \overline{1})\},
$$

and equations of $\left(10^{\prime}\right)$ by the elements of

$$
L_{4,(1,1)}^{2}=\{(0,0),(1,0),(2,0),(3,0),(0,1),(3,1),(0,2),(3,2)\} .
$$

Since these sets are disjoint, the points indexed by $(x, y)$ and $(\bar{x}, \bar{y})$ are seen as distinct.

The determinant of the system $\left(9^{\prime}\right)-\left(10^{\prime}\right)$ has the form

$$
\operatorname{det} A_{2}=\left|\begin{array}{cccccccccccc}
1 & 0 & 0 & 0 & 0 & 0 & 0 & 0 & 0 & 0 & 0 & 0 \\
0 & 1 & 0 & 0 & 0 & 0 & 0 & 0 & 0 & 0 & 0 & 0 \\
0 & 0 & 1 & 0 & 0 & 0 & 0 & 0 & 0 & 0 & 0 & 0 \\
0 & 0 & 0 & 1 & 0 & 0 & 0 & 0 & 0 & 0 & 0 & 0 \\
0 & 0 & 0 & 0 & 1 & 0 & 0 & 0 & 0 & 0 & 0 & 0 \\
c_{00} & c_{10} & c_{20} & 0 & c_{01} & c_{11} & c_{21} & 0 & 0 & 0 & 0 & 0 \\
0 & c_{00} & c_{10} & c_{20} & 0 & c_{01} & c_{11} & c_{21} & 0 & 0 & 0 & 0 \\
0 & 0 & 0 & 0 & 0 & 0 & 0 & 1 & 0 & 0 & 0 & 0 \\
& & & & & & & & & & & \\
0 & 0 & 0 & 0 & 0 & 0 & 0 & 0 & 1 & 0 & 0 & 0 \\
0 & 0 & 0 & 0 & c_{00} & c_{10} & c_{20} & 0 & c_{01} & c_{11} & c_{21} & 0 \\
0 & 0 & 0 & 0 & 0 & c_{00} & c_{10} & c_{20} & 0 & c_{01} & c_{11} & c_{21} \\
0 & 0 & 0 & 0 & 0 & 0 & 0 & 0 & 0 & 0 & 0 & 1
\end{array}\right| .
$$


Denote by $\|A\|_{\infty}=\max _{i} \sum_{j=1}^{n}\left|a_{i j}\right|$ the maximum norm of the matrix $A$ and by $R_{i}(A)=$ $\left|a_{i i}\right|-\sum_{j \neq i}\left|a_{i j}\right|, i=1,2, \ldots, n$ the value of the diagonal dominance in each row. Set also $R_{*}(A)=\min _{1 \leqslant i \leqslant n} R_{i}(A)$, if $R_{*}(A) \geqslant 0$ then $A$ is a diagonally dominant matrix (see [16]).

Proof of Theorem 1. The determinant of the matrix $A_{p}$ has either 1 or the coefficient $c_{\beta}$ is on the main diagonal. If the condition (8) is fulfilled the matrices $A_{p}$ are diagonally dominant, so $\operatorname{det} A_{p} \neq 0$ (see, e.g. [17]) and the problem (9)-(10) has a unique solution.

To prove stability we need to estimate the norm of the matrix $A_{p}^{-1}$, which is the inverse for the matrix $A_{p}$ of the subsystem (9) - (10).

For any $p \in \mathbb{Z}_{+}$the values of diagonal dominance in lines numbered by $\left(\mu_{1}, \mu_{2}\right) \in$ $L_{K, \beta}^{p}$ are equal to $R_{\left(\mu_{1}, \mu_{2}\right)}\left(A_{p}\right)=1$, and in lines numbered by $(x, y) \in \Pi_{K}^{p}$ they are $R_{(x, y)}\left(A_{p}\right)=\left|c_{\beta m}\right|-\sum_{(i, j) \neq(\beta, m)}\left|c_{i j}\right|$. All these quantities are independent of $p$. Thus, $R_{*}\left(A_{p}\right)=\min \left\{1,\left|c_{\beta m}\right|-\sum_{(i, j) \neq(\beta, m)}\left|c_{i j}\right|\right\} \neq 0$. For a diagonally dominant matrix $A_{p}$ there is an estimate $([16])$

$$
\left\|A_{p}^{-1}\right\|_{\infty} \leqslant \frac{1}{R_{*}\left(A_{p}\right)}
$$

Consider the vector $f_{p}$ with coordinates $f(x, y),(x, y) \in \Pi_{K}^{\beta}$ and the vector $h_{p}$ with coordinates $g(x, y), \varphi\left(\mu_{1}, \mu_{2}\right)$, where $(x, y) \in L_{K}^{p},\left(\mu_{1}, \mu_{2}\right) \in L_{K, \beta}^{p}$. The system (9)-(10) can be written in the form $A_{p} f_{p}=h_{p}$, where $\operatorname{det} A_{p} \neq 0$, from here we find $f_{p}=A_{p}^{-1} h_{p}$. Now we estimate the norm of $f_{p}$ taking into account (11):

$$
\begin{gathered}
\left\|f_{p}\right\|_{\infty}=\left\|A_{p}^{-1} h_{p}\right\|_{\infty} \leqslant\left\|A_{p}^{-1}\right\|_{\infty}\left\|h_{p}\right\|_{\infty} \leqslant \frac{1}{R_{*}\left(A_{p}\right)}\left\|h_{p}\right\|_{\infty} \leqslant \\
\leqslant M\left(\|g(x, y)\|_{\infty}+\|\varphi(x, y)\|_{\infty}\right) .
\end{gathered}
$$

Since the inequality is valid for all $p,\|f\|_{\infty} \leqslant M\left(\|g(x, y)\|_{\infty}+\|\varphi(x, y)\|_{\infty}\right)$. Thus, the problem (2)-(3) is stable.

Consider an application of the obtained results to the problem (5)-(6). Since for the coefficients of the polynomial difference operator we have $1 \geqslant|a|+|a|+|2 a+1|, a=-\frac{\gamma}{1+2 \gamma}$, the condition of diagonal dominance (8) in Theorem 1 is fulfilled, therefore the problem of (5)-(6) has a unique solution and is stable.

Let us give an example showing that the condition (8) of Theorem 1 is sufficient but not necessary. Consider the approximation of the problem (4) by a three-layer scheme of the form

$$
\begin{gathered}
\Theta \frac{u_{i}^{m+2}-u_{i}^{m+1}}{\tau}+(1-\Theta) \frac{u_{i}^{m+1}-u_{i}^{m}}{\tau}-\sigma_{1} \frac{u_{i+1}^{m+2}-2 u_{i}^{m+2}+u_{i-1}^{m+2}}{h^{2}}- \\
-\left(1-\sigma_{1}-\sigma_{2}\right) \frac{u_{i+1}^{m+1}-2 u_{i}^{m+1}+u_{i-1}^{m+1}}{h^{2}}-\sigma_{2} \frac{u_{i+1}^{m}-2 u_{i}^{m}+u_{i-1}^{m}}{h^{2}}=0, i=1,2, \ldots, K .
\end{gathered}
$$

In the case of a purely implicit scheme, namely when $\sigma_{1}=1, \sigma_{2}=0, \Theta=\frac{3}{2}$, the scheme (12) can be written as $\frac{3}{2} \frac{u_{i}^{m+1}-u_{i}^{m}}{\tau}-\frac{1}{2} \frac{u_{i}^{m+1}-u_{i}^{m}}{\tau}-\frac{u_{i+1}^{m+2}-2 u_{i}^{m+2}+u_{i-1}^{m+2}}{h^{2}}=0$. Denote $\gamma=\frac{\tau}{h^{2}}$, then

$$
u_{i}^{m+2}-\frac{2 \gamma}{3+4 \gamma} u_{i+1}^{m+2}-\frac{2 \gamma}{3+4 \gamma} u_{i-1}^{m+2}-\frac{4}{3+4 \gamma} u_{i}^{m+1}+\frac{1}{3+4 \gamma} u_{i}^{m}=0 .
$$


In our notation the difference operator has the form $P\left(\delta_{1}, \delta_{2}\right)=\delta_{1} \delta_{2}^{2}-\frac{2 \gamma}{3+4 \gamma} \delta_{1}^{2} \delta_{2}^{2}-\frac{2 \gamma}{3+4 \gamma} \delta_{2}^{2}-$ $\frac{4}{3+4 \gamma} \delta_{1} \delta_{2}+\frac{1}{3+4 \gamma} \delta_{1}$. If we denote $u_{i}^{m}=f(i, m)$, then the Cauchy problem for this scheme takes the form

$$
\begin{gathered}
P\left(\delta_{1}, \delta_{2}\right) f(x, y)=0, \quad 0<x<K-1, \quad y=0,1, \ldots, \\
\varphi(x, y)=\left\{\begin{array}{l}
f(0, y), y=0,1, \ldots, \\
f(K, y), y=0,1, \ldots, \\
f(x, 0), x=1,2, \ldots, K-1, \\
f(x, 1), x=1,2, \ldots, K-1 .
\end{array}\right.
\end{gathered}
$$

The condition (8) of Theorem 1 does not hold, since $\frac{2 \gamma}{3+4 \gamma}+\frac{2 \gamma}{3+4 \gamma}+\frac{4}{3+4 \gamma}+\frac{1}{3+4 \gamma}=$ $\frac{4 \gamma+5}{3+4 \gamma}>1$, however it is known that this problem is stable (see, e.g. [4]).

The work of the second author was financed by a grant of the President of the Russian Federation for state support of leading scientific schools (no. NSh-9149.2016.1).

\section{References}

[1] A.O.Gel'fond, The calculus of finite differences, Moscow, KomKniga, 2006 (in Russian).

[2] M.Bousquet-Melou, M.Petkovšek, Linear recurrences with constant coefficients: the multivariate case, Discrete Mathematics, 225(2000), 51-75.

[3] E.K.Leinartas, Multiple Laurent series and fundamental solutions of linear difference equations, Sib. Math. J., 48(2007), no. 2, 335-340.

[4] A.A.Samarskii, Introduction to the theory of differential circuits, Moscow, Nauka, 1971 (in Russian).

[5] V.S.Ryaben'kii, On the stability of difference equations, Moscow, Gos. izd. tekhnikoteoreticheskoy literatury, 1956 (in Russian).

[6] M.S.Rogozina, Stability of multilayer finite difference schemes and amoebas of algebraic hypersurfaces, Journal of Siberian Federal University. Mathematics \& Physics, 5(2012), no. 2, 256-263 (in Russian).

[7] R.Izerman, Digital Control Systems, Springer-Verlag, 1981.

[8] A.K.Tsikh, Conditions for absolute convergence of the Taylor coefficient series of a meromorphic function of two variables, Mathematics of the USSR-Sbornik, 74(1993) no. 2, 337-360.

[9] M.V.Fedoruk, Asymptotics: integrals and series, Moscow, Nauka, 1987 (in Russian).

[10] M.S.Rogozina, Stability of multilayer inhomogeneous difference schemes and amoebas of algebraic hypersurfaces, Vestnik SibGAU, 49(2013), no. 3, 95-99 (in Russian).

[11] E.K.Leinartas, The stability of the Cauchy problem for the homogeneous difference operator and the amoeba of characteristic set, Sib. Math. J., 52(2011), no. 5, 1087-1095. 
[12] M.S.Rogozina, On the solvability of the Cauchy problem for a polynomial difference operator, Vestnik Novosibirskogo Gosudarstvennogo Universiteta. Seriya Matematika, Mekhanika, Informatika, 14(2014), no. 3, 83-94 (in Russian).

[13] E.K.Leinartas, M.S.Rogozina, Solvability of the Cauchy problem for a polynomial difference operator and monomial bases for the quotients of a polynomial ring, Sib. Math. J., 56(2015), no. $1,92-100$.

[14] M.S.Rogozina, On the correctness of polynomial difference operators, Journal of Siberian Federal University. Mathematics \& Physics, 8(2012), no. 4, 437-441.

[15] L.Hörmander, An introduction to complex analysis in several variables, 3 Ed., North Holland, 1990.

[16] Yu.S.Volkov, V.L.Miroshnichenko, Norm estimates for the inverses of matrices of monotone type and totally positive matrices, Sib. Math. J., 50(2009), no. 6, 982-987.

[17] S.P.Sharyi, A course of computational methods, Novosibirsk, Novosibirsk Univ., 2012.

\section{Корректность двумерной задачи Коши для полиномиального разностного оператора с постоянными коэффициентами}

Марина С. Апанович

Красноярский государственный медицинский университет Партизана Железняка, 1, Красноярск, 660022

Россия

Евгений К. Лейнартас

Институт математики и фундаментальной информатики Сибирский федеральный университет Свободный, 79, Красноярск, 660041

Россия

$\overline{\text { Исследуется корректность задачи Коши для полиномиального разностного оператора. Доказано }}$ легко проверяемое достаточное условие корректности двумерной задачи Коши для оператора с постоянными коэффициентами.

Ключевые слова: полиномиальный разностный оператор, задача Коши, корректность. 